\title{
Aprendizaje lingüístico e inclusión social: combinación del enfoque comunicativo y el enfoque por tareas.
}

\author{
BORJA TALENS COMPANY \\ Universidad de Lleida \\ btc2@alumnes.udl.cat
}

\begin{abstract}
Resumen: La presente propuesta de experiencia práctica aspira a compartir los buenos resultados conseguidos en un curso de español como lengua extranjera dirigido a inmigrantes realizado en "L'Espai d'Inclusió i Formació Casc Antic" (Eicascantic Barcelona), en el que se combinó el enfoque comunicativo con el enfoque por tareas con los objetivos de mejorar el nivel lingüístico de los alumnos y de favorecer su inclusión social en su nuevo contexto. Para ello, se creó el material sociolingüístico del nivel A2 del MCERL Conociendo y se aplicó en un curso intensivo de 54 horas de duración llevando a cabo, a posteriori, un análisis de los resultados obtenidos. Todo ello se realizó teniendo en mente el objetivo principal de la entidad: promover la inclusión social de personas jóvenes y adultas de diversos orígenes mediante iniciativas de formación y participación que faciliten el ejercicio de una ciudadanía activa y responsable, la convivencia y la cohesión social.
\end{abstract}

Palabras clave: Aprendizaje L2, inmigración, inclusión social, enfoque comunicativo, enfoque por tareas, prácticas comunicativas.

Abstract: The present proposal of practical experience aims to share the good results achieved in a course of Spanish as a foreign language for immigrants carried out in "L'Espai d'Inclusió i Formació Casc Antic" (Eicascantic) in which the communicative approach and the task-based approach were combined in order to fulfil objectives of improving the linguistic level of students and promoting their social inclusion in their new context. To this end, the sociolinguistic material of level A2 of the MCERL "Conociendo" was created and applied in an intensive course of 54 hours duration, carrying out, afterwards, an analysis of the results obtained. All this was done with the main objective of the organization in mind: to promote the social inclusion of young people and adults of different origins through training and participation initiatives that facilitate the exercise of active and responsible citizenship, coexistence and social cohesion.

Keywords: L2 learning, immigration, social inclusion, communicative approach, taskbased approach, communicative practices. 


\section{Introducción}

La presente propuesta práctica corresponde al TFM del Máster en Lenguas Aplicadas de la Universidad de Lleida titulado Observación, creación de materiales y aplicación práctica en las clases de español A2 de "L'Espai d'Inclusió $i$ Formació Casc Antic" (Eicascantic) en el que se realizó un periodo de prácticas en la asociación Eicascantic de Barcelona. Como parte de las prácticas era necesario detectar una necesidad de la asociación y hacer una propuesta de mejora que, en este caso, fue crear los materiales de referencia para los cursos intensivos del nivel A2 que la asociación ofrece durante el verano pero que, a su vez, fuesen fáciles de complementar para los cursos de larga duración que se ofrecen en invierno. Además, se intentó dar solución a una de las problemáticas previamente detectadas por la asociación: la dificultad que presentan los alumnos para trasferir los contenidos lingüísticos trabajados en las clases a un contexto de comunicación real. Así, se complementó el material con prácticas comunicativas y se aplicó en el curso de verano de julio del 2016. Presentamos a continuación todo el proceso desde su comienzo con las observaciones iniciales, pasando por el proceso de creación de materiales y terminando con su aplicación y el análisis de los resultados obtenidos.

\section{Contextualización de la entidad}

"L’Espai d'Inclusió i Formació Casc Antic" (Eicascantic) es una asociación sin ánimo de lucro que tiene 30 años de experiencia en la acogida, la formación, el diálogo y la participación asociativa y ciudadana para favorecer la inclusión socio-laboral de la población joven y adulta, sin hacer diferencias de edad, género, origen o nivel económico. Además, es un espacio que representa fielmente la multiculturalidad existente en nuestra sociedad, siendo este un elemento clave para la socialización de las personas inmigradas que encontramos en la entidad.

De entre todos los proyectos que se llevan a cabo en la asociación, nuestro trabajo se enmarca en el de la intervención socioeducativa para la inclusión social que se estructura en cuatro ejes de actuación: acogida, mediación, formación y participación. Dicho proyecto parte de una propuesta de acogida formativa en competencias básicas inclusiva para personas adultas de diferentes procedencias que se concreta en un servicio de inmersión sociolingüística y de formación instrumental básica.

A partir de los cuatro ejes de la entidad, se busca propiciar un espacio en donde personas en riesgo de exclusión (como pueden ser las inmigradas y/o con un bajo nivel de formación) puedan aprender y adquirir herramientas desde el ámbito formativo para mejorar sus condiciones económicas, sociales y culturales y llegar a ser ciudadanos activos en nuestra sociedad.

El servicio atiende aproximadamente a 600 personas al año y contempla la formación inicial en lenguas vehiculares (castellano y catalán), conocimiento del entorno, formación instrumental básica en dos niveles (alfabetización y neolectura) e iniciación a la informática. Por otra parte, desde la asociación se realizan actividades culturales para promover el ocio y la convivencia para la inclusión social, buscando potenciar la participación de las personas que acuden a la entidad. 


\section{Explicación de las líneas de actuación del trabajo}

Las actuaciones que se llevaron a cabo durante las prácticas del TFM fueron:

-Realización de un período de observación de las clases de Eicascantic en diferentes niveles para poder conocer desde dentro el funcionamiento de la asociación.

-Creación de los materiales (libro del alumno y libro del profesor) para un curso intensivo de 54 horas que repasase gran parte de los contenidos gramaticales, léxicos, pragmáticos y socioculturales del nivel A2.

-Creación de una serie de prácticas comunicativas en contextos reales de comunicación que complementasen a las unidades del libro y que sirviesen para que los alumnos aplicasen los conocimientos trabajados en clase en un contexto real de comunicación, mejorando así el conocimiento de su entorno inmediato y favoreciendo su inclusión social.

-Impartición del curso intensivo para probar el material en el aula y comprobar su efectividad.

-Realización de un breve análisis de resultados de la mejora del nivel lingüístico de los alumnos basándose en las pruebas contraste creadas.

\section{Desarrollo de la experiencia}

A partir del periodo de observación, desde el que se llegó a conocer en profundidad la asociación y su funcionamiento, se inició el proceso de creación del material del nivel A2 del MCERL Conociendo. De dicha observación se dedujeron las siguientes consideraciones que guiaron el proceso de creación del material:

1. Debía englobar todas o la mayoría de las funciones, los contenidos léxicos, gramaticales y funcionales del nivel A2.

2. La metodología tenía que estar basada en los enfoques comunicativos proporcionando abundantes muestras auténticas de lengua.

3. Los contenidos debían estar adaptados a los intereses de los alumnos así como reflejar su realidad inmediata: el barrio, la ciudad, Cataluña, España.

4. La asistencia al curso no era obligatoria, por lo que debía ser un material atractivo y funcional con un formato y unas actividades que motivasen a los alumnos a asistir diariamente.

5. Al haber observado la multiculturalidad presente en todas las aulas, con cada conocimiento cultural que se presentase se tenía que pedir a los alumnos que lo contrastasen con su propia cultura y que lo compartiesen con el resto de la clase para propiciar un diálogo intercultural y el reconocimiento de pertenencia a una sociedad multicultural.

6. Por las características del alumnado y por sus necesidades (inclusión sociolaboral), e intentando relacionar los materiales con los cursos de iniciación a la informática que lleva a cabo la asociación, el material tenía que incluir actividades en las que se usaran las nuevas tecnologías disponibles en el centro (en este caso, la sala de ordenadores de la asociación).

7. En el libro, de acuerdo con el Plan Curricular del Instituto Cervantes (2007), debían aparecer los siguientes cinco componentes: gramatical, pragmático- 
discursivo, nocional (nociones generales y específicas), cultural y el de aprendizaje.

8. Se crearían unas prácticas comunicativas en contextos reales controlados y no controlados relacionadas con los contenidos trabajados para afianzarlos y darles a los alumnos la posibilidad de practicarlos en contextos reales de comunicación.

\section{CONOCIENDO}

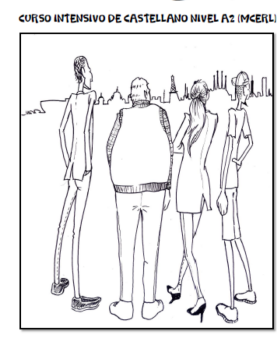

HBRODEL ALUMNO

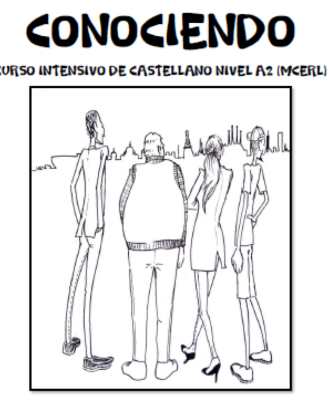

LURO DEL PROFESOR

Imagen 1: Conociendo: Libro del alumno y libro del profesor creados para Eicascantic ${ }^{l}$

\subsection{Descripción del material}

\subsubsection{Características del alumnado al que está destinado}

El curso está dirigido a personas jóvenes o adultas que han inmigrado a nuestro país, que disponen de un nivel de formación muy diverso (desde niveles bajos o nulos hasta medios o superiores en su país de origen) que necesitan adquirir las competencias comunicativas básicas en la lengua española para aumentar sus posibilidades de integración sociolaboral en el nuevo entorno. Sin embargo, estos perfiles no son exclusivos ni excluyentes, ya que el curso puede aplicarse a personas extranjeras con otras características formativas y sociales.

Concretamente, al curso asistieron 13 alumnos de entre 22 y 50 años con diferentes grados de formación previa que abarcaban desde la escolarización obligatoria a la formación universitaria en sus países de origen. Los alumnos provenían de China, Ucrania, Rusia, Irán, Marruecos, Polonia, Italia, Nueva Zelandia e Irlanda.

\subsubsection{Justificación: enfoques metodológicos}

El material se creó siguiendo el enfoque comunicativo centrado en el alumno, en el que se trabajan las cuatro destrezas poniendo especial énfasis en la comprensión y en la expresión oral. Con este modelo didáctico se pretende capacitar al estudiante para una comunicación real -no solo en la vertiente oral, sino también en la escrita- con otros hablantes de la LE, es decir, se pretende que los alumnos adquieran una competencia comunicativa suficiente para su inclusión en el nuevo contexto social en el que viven. Tal y como apuntan Canale y Swain (1980), dicha competencia comunicativa engloba un conocimiento subyacente que contiene el conocimiento gramatical de la lengua y, a su

\footnotetext{
${ }^{1}$ El material Conociendo está registrado en el Registro de la Propiedad Intelectual con la referencia $02 / 2017 / 98$
} 
vez, la capacidad de usarla en situaciones concretas de comunicación de manera adecuada.

Pilar García (1997: 358) define el espacio comunicativo como «el ámbito en que el inmigrante entra en contacto estableciendo intercambios comunicativos con todos los elementos que lo configuran social, lingüística, pragmática, cultural y estratégicamente».

En este caso, los contextos o espacios comunicativos en los que el inmigrante se mueve diariamente son España, Barcelona y, más concretamente, el barrio del Casc Antic. Por consecuencia, los exponentes socioculturales y de conocimiento del entorno se centraron en dichos ámbitos.

Teniendo en cuenta que los alumnos del curso, al empezar las clases, ya se encontraban viviendo en Barcelona por un periodo de entre 1 y 6 meses, se sobreentendió que, teniendo un nivel A1.1/A2, ya habrían establecido relaciones con diferentes interlocutores y tendrían cierta familiaridad con los tipos de códigos y registros que se utilizan en las diferentes situaciones de comunicación o espacios comunicativos. Asimismo, habrían circulado por las calles, se moverían diariamente en transporte público y conocerían la ciudad (o al menos su entorno inmediato). Consecuentemente, habrían utilizado la lengua española en contextos reales. Sin embargo, coincidiendo con la problemática detectada por Andrea Menegotto (1998) en los cursos intensivos de inmersión lingüística diseñados para la Universidad de Mar del Plata, uno de los problemas principales que presentan los alumnos de la asociación es transferir las habilidades lingüísticas trabajadas en clase a un contexto real de comunicación. Para subsanarla, la autora creó cursos de inmersión lingüística basados en el enfoque por tareas que permitiesen a los alumnos aplicar la lengua en contextos reales de comunicación y, en un intento de obtener los buenos resultados del proyecto, decidimos adaptar el modelo de estructuración del curso y la metodología utilizada a las necesidades de la asociación.

En las interacciones orales «el estudiante debe cumplir simultáneamente distintas operaciones cognitivo-lingüísticas, como el procesamiento de la información que recibe de su interlocutor, la automatización de ciertas operaciones lingüísticas para expresarse, la organización de la información, la adaptación de la producción al tipo de discurso específico, la negociación del sentido y la resolución» (Di Franco, 2006: 471). Por este motivo, se optó por complementar los materiales creados con prácticas comunicativas en contextos reales de comunicación para favorecer la práctica y la aplicación directa de los contenidos trabajados durante el curso en contextos reales que, a su vez, favoreciesen directamente la inclusión y la participación activa de los alumnos en su nuevo contexto social.

Al adaptar la estructuración del curso y su metodología al contexto de la asociación, se hizo patente que no podía basarse exclusivamente en el trabajo por tareas debido a la falta de recursos humanos que supervisasen las prácticas de los alumnos y al poco tiempo disponible para su implementación. Por ese motivo las prácticas comunicativas, aunque guardan cierta relación con el enfoque por tareas, no pueden describirse como tal ya que contrariamente a dicho enfoque, en las prácticas creadas primero se lleva a cabo un análisis de la lengua, en sus distintos niveles de descripción, obteniendo los inventarios lingüísticos (de vocabulario, de estructuras morfosintácticas, de nociones y funciones) mediante el trabajo de los exponentes presentados en el libro, a partir de cuya interiorización los alumnos pueden realizar las prácticas. En dicha fase, las tareas van precedidas de un análisis del contexto en el que se va a establecer la comunicación para 
determinar qué expresiones y fórmulas deberán utilizar para conseguir el objetivo propuesto.

El punto en común con el enfoque por tareas es que el input presentado en el libro y las actividades realizadas en el aula utilizan muestras representativas de comunicación que se llevan a cabo fuera de ella y que, además, las tareas están diseñadas para satisfacer necesidades inmediatas de los estudiantes que previamente han sido detectadas por el docente. El curso se estructura, pues, en un $75 \%$ de clase en el aula y un $25 \%$ de prácticas en contextos reales y, también, simulados.

\subsubsection{Contenidos}

\section{a) Gramaticales, fonéticos y prosódicos}

La premisa fundamental en la que nos basamos para presentar y trabajar los contenidos gramaticales fue la siguiente:

La gramática, entendida como el código formal, se enseñará subsidiariamente de los objetivos comunicativos que se establezcan en la programación. Dicho de otro modo, conjugar un verbo, acumular léxico descontextualizadamente, saber las reglas de formación de palabras, conocer el metalenguaje gramatical, etc., no serán los objetivos que vamos a perseguir, sino que se convertirán en objetivos dependientes de los objetivos comunicativos.

(López, 1995: 242)

Por tanto, la metodología que se lleva a cabo está basada en el enfoque comunicativo más centrado en la comprensión y la expresión oral aunque sin dejar de lado la expresión y la comprensión escrita, ya que el objetivo de muchos alumnos de Eicascantic es presentarse al examen DELE.

Teniendo en cuenta las necesidades inmediatas de aplicación de la lengua en contextos reales de comunicación, tanto las actividades como las normas gramaticales se abordan desde una perspectiva funcional para conseguir la «interiorización y automatización gramática objeto (en adelante $\mathrm{GO}^{2}$ ) en el aula a través de muestras auténticas o verosímiles y la realización de prácticas comunicativas apropiadas a cada caso y nivel de competencia» tal y como aconseja Ventura Salazar (1992:15). Presentando los mecanismos gramaticales desde la perspectiva de la comunicación, obtenemos «una adecuada estructura conceptual que permite abarcar fenómenos muy dispares» (Gómez del Estal, 2008: 88) que, además, nos ayudará a relacionar los contenidos gramaticales con los léxicos, morfológicos, sintácticos y pragmáticos propios de las situaciones que se le presentan al alumno.

Es por eso por lo que los contenidos gramaticales se presentan de manera natural con un objetivo comunicativo práctico siguiendo el método inductivo explícito para que el alumno interiorice las reglas gramaticales de forma consciente, reflexionando sobre su aprendizaje y formulando hipótesis de uso mediante el input que se le presenta para, posteriormente, comprobarlas o reformularlas adquiriendo un conocimiento gramatical significativo y funcional para un uso competente.

\footnotetext{
${ }^{2}$ Salazar (1992) se refiere al funcionamiento interno de una lengua, es decir, a la competencia de que dispone un hablante en dicha lengua como gramática objeto (GO).
} 
El material carece de contenidos fonéticos y prosódicos específicos y explícitos puesto que se decidió ir corrigiendo y explicando los posibles errores a medida que fuesen surgiendo en el aula. De esta manera, se detectaron en algunos alumnos errores que fueron explicados $\mathrm{y}$, el día siguiente a su explicación, se les proporcionó a dichos alumnos materiales para que pudiesen trabajar en los errores detectados y subsanarlos.

\section{b) Léxicos}

El léxico y el vocabulario trabajados en el curso engloban todas las nociones específicas del Plan Curricular del Instituto Cervantes, viéndose ampliados tanto la exposición como el aprendizaje directo del léxico, de colocaciones y de unidades fraseológicas en todas las unidades a través de la construcción de redes asociativas basadas en la denotación y la connotación del léxico en los diferentes contextos en los que se desenvuelven diariamente.

Así, el vocabulario y el léxico general no se presentan predeterminados sino que se inducen a partir de imágenes y de situaciones reales de comunicación y se interiorizan mediante actividades lúdicas, creativas, asociativas y de práctica sistemática que «faciliten tanto la comprensión, retención y utilización de las unidades léxicas como desarrollar estrategias de aprendizaje y comunicación que permitan al alumno aumentar su vocabulario» (Hayas, 2009: 363).

Para ello, la mayoría de las actividades de léxico se abordan desde un enfoque creativo (Hayas, 2009) partiendo de los conocimientos previos del alumnado que son puestos en común mediante lluvias de ideas o preguntas formuladas en base a la observación de imágenes o textos. Siguiendo este método, el profesor es libre de ampliar el vocabulario trabajado, adaptándolo al nivel y a las necesidades diversas del alumnado asistente al curso.

En consecuencia, no se especifica una cantidad determinada de vocabulario que se trabaja en cada tema, siendo especialmente relevante comentar que, desde dicho enfoque, previsiblemente, se trabajará vocabulario que excede el propuesto para el nivel A2. Es por este motivo por el que, al final de cada tema, se habilita un espacio para que los alumnos anoten el nuevo vocabulario, las expresiones y el léxico relacionado que han aprendido y que consideren de utilidad para su día a día.

\section{c) Socioculturales}

Los intereses de los alumnos del curso están muy ligados al conocimiento del entorno y de la cultura por lo que, como parte del proyecto sociolingüístico de Eicascantic, en el curso se integran abundantes conocimientos relativos a la comunidad hispanohablante española aunque más centrados en la ciudad de Barcelona, en sus habitantes y en las ofertas culturales y lúdicas que presenta la ciudad. Tal y como defiende Starc:

La vida social se desarrolla a partir de las interacciones comunicativas entre los hablantes de una lengua. En las interacciones, se pone a prueba la competencia comunicativa de los hablantes, es decir, su capacidad para comportarse de manera apropiada y eficaz en diferentes contextos. El contexto social es uno de los factores más influyentes en el proceso de adquisición de una lengua. De ahí la importancia de considerar a la lengua en su contexto social incorporando el componente sociolingüístico de la comunicación en el proceso de aprendizaje. 
Tomando esta afirmación como punto de partida y siendo conocedores de las necesidades inmediatas de uso lingüístico de los alumnos asistentes al curso, los conocimientos socioculturales y del entorno que se trabajan explícitamente en cada tema por su relación con los contenidos trabajados son: saludos formales e informales, personajes famosos del mundo, las lenguas que se hablan en España, procedimientos para empadronarse y solicitar la TSI, los centros de salud, la biografía y obra de Antonio Gaudí, diversos buscadores de trabajo online (Servei d'ocupació de Catalunya, LinkedIn e Infojobs), las nóminas, los trayectos de autobuses y metro de Barcelona, el uso de Google maps, las ofertas culturales y de ocio de Barcelona y las comidas típicas de España.

\subsubsection{Prácticas comunicativas}

Se diseñaron tres prácticas relacionadas directamente con los contenidos trabajados. Debido a la falta de recursos personales, en todas las prácticas no se pudieron hacer intercambios lingüísticos reales, sin embargo, se relacionaron directamente con situaciones comunicativas a las que los alumnos deberán hacer frente como parte activa del proceso de inclusión en su nuevo contexto social.

La primera práctica consistió en visitar y familiarizarse con el portal web www.amigosbarcelona.com, crearse un perfil y publicar un anuncio. Su objetivo principal fue dotar a los alumnos de los recursos necesarios para relacionarse con diversos agentes sociales (nativos o no) que viviesen en su mismo contexto (Barcelona) y que, como resultado de dicha interacción, pudiesen practicar la lengua o realizar actividades lúdicas y de práctica lingüística en su entorno inmediato, favoreciendo así su inclusión en él. Esta práctica está relacionada con los temas 1 y 2.

El portal web es una red social para conocer gente en Barcelona de forma gratuita en un ambiente de consumo colaborativo. Los usuarios de la página organizan semanalmente excursiones y actividades gratuitas, muchas de ellas aprovechando los recursos de los que dispone la ciudad de Barcelona. Aparte de estas actividades que se publican en la página de inicio, existen diversos grupos creados por áreas de interés en los que se puede buscar pareja, buscar compañeros/as para practicar hobbies (deportes, danza, cultura, religión, excursiones, series, películas...), buscar gente en tu misma situación (en la página se llama "expatriados en Barcelona") o hacer intercambios de servicios e intercambios lingüísticos (que era la actividad que más nos interesaba). En estos intercambios lingüísticos se crean grupos para practicar lenguas y se ofertan parejas lingüísticas en las que se ofrece enseñar o practicar oralmente una lengua a cambio de otra.

La segunda práctica consistió en navegar por el portal www.infojobs.net, buscar una oferta de trabajo que les interese, escribir una carta de presentación, traducir su CV al español y enviarlo a la dirección proporcionada (en este caso, al ser una práctica simulada, se enviaba a la dirección del profesor que le contestaba el correo con las correcciones y sugerencias pertinentes).

Esta práctica está directamente relacionada con el tema 3 y su objetivo principal fue que los alumnos tuviesen preparado su currículum vitae y una carta de presentación correcta en español para cuando lo pudiesen necesitar.

Las dos primeras prácticas se llevaron a cabo individualmente y fueron supervisadas en todo momento por el docente. 
La tercera y última práctica se llevó a cabo en un contexto real y no se trató de una simulación. Al ser la última práctica del curso, el profesor ya conocía a los alumnos y, por lo tanto, se decidió crear prácticas que estuviesen relacionadas con los intereses individuales de cada uno de ellos. El objetivo general de la práctica fue que los alumnos afrontasen una situación real de comunicación con un objetivo predeterminado, analizando con anterioridad los recursos lingüísticos necesarios para llevar a cabo la tarea con éxito. Para ello, se les proporcionó un documento en el que cada alumno tenía que rellenar: las fórmulas de cortesía que iba a utilizar, las preguntas que debía formular y, finalmente, la información recogida. El día siguiente a las prácticas, los alumnos expusieron al resto de la clase la información recabada. Las prácticas fueron:

- Ir a una herboristería y preguntar por remedios naturales para el dolor de espalda. - Ir a la Oficina de Atención Ciudadana más cercana y preguntar por la documentación necesaria para solicitar el empadronamiento.

- Ir al mercado de Santa Catalina y pedir presupuestos en diversas tiendas para contratar un catering para 10 personas.

- Ir a la biblioteca más cercana, hacerse el carnet de socio y preguntar por los servicios a los que se tendría acceso.

- Ir al Centro Cultural del Born y preguntar por las ofertas culturales y de ocio del mes de agosto.

- Ir al Museo del Chocolate y preguntar por el precio de las visitas en grupo.

- Ir a una escuela privada de idiomas e informarse sobre los cursos intensivos del nivel A2 que ofertan.

- Ir al centro cívico más cercano y preguntar qué es y qué actividades realizaban.

- Ir al "Casal del Barri" e informarse sobre qué es, cómo funciona y qué servicios ofrece.

- Visitar la "Xarxa de Consum Solidari” e informarse sobre cómo funciona.

\subsubsection{Evaluación}

Dado que la asociación ofertaba los cursos de revisión y afianzamiento de los contenidos relativos al nivel A1 y A2, para poder clasificar a los alumnos según su nivel, se les aplicó una prueba oficial del Instituto Cervantes del nivel A2 del año 2010 a través de la que se evaluaba su nivel competencial en comprensión auditiva, comprensión lectora, expresión escrita y expresión oral.

Es oportuno remarcar que el objetivo de la asociación no es conseguir unos resultados concretos, individuales y/o globales del grupo/clase, sino proporcionar el servicio de enseñanza lingüística como parte fundamental del proceso de inclusión social de personas inmigradas. Por este motivo, la realización de exámenes no es una práctica habitual en la asociación y optan por otros procedimientos evaluativos como tomar actividades de los materiales como pruebas evaluativas, rellenar fichas de observaciones de cada alumno y, aleatoriamente, pasar pruebas contraste para apreciar su evolución.

En el caso del presente curso, para poder valorar la eficacia y funcionalidad de los materiales creados y la evolución de los alumnos, se decidió que al final del curso se les pasaría la misma prueba (sin haberles mostrado las correcciones ni el resultado de la primera) para poder tener al menos una herramienta comparativa que nos permitiese obtener datos con un cierto carácter objetivo (prueba contraste). Dichos datos nos 
ayudaron, por un lado, a observar la mejora individual de cada alumno y, por otro, a comprobar si los materiales eran funcionales y permitían dicha mejora.

\section{Resultados y trabajo futuro}

Presentamos un gráfico comparativo que muestra el contraste entre los resultados de las pruebas iniciales y finales, en cuanto al nivel competencial de la clase, diferenciados por destrezas para comentarlos a continuación:

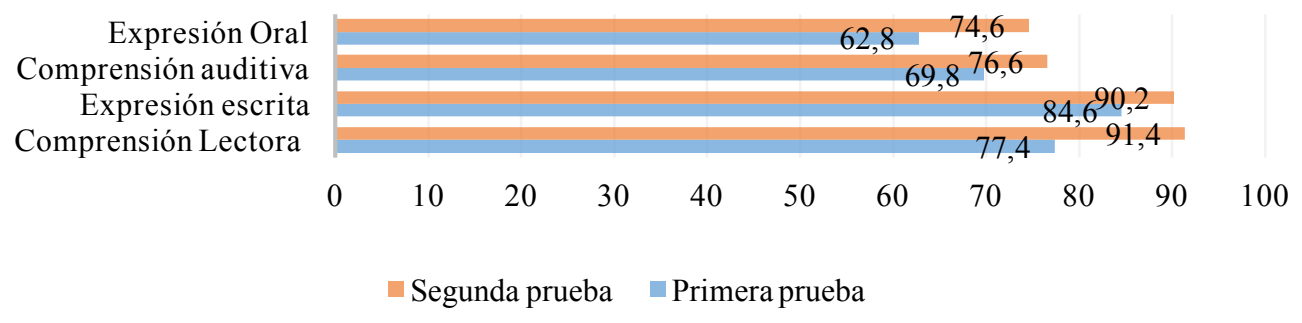

Gráfico 1: Comparación de los porcentajes de los resultados globales por competencias

A partir del análisis de los porcentajes, podemos afirmar que los resultados del grupo/clase mejoraron un 14\% en comprensión lectora, un 5.6\% en expresión escrita, un $6,8 \%$ en comprensión auditiva y un $11,8 \%$ en expresión oral. La media de la clase, que inicialmente era de un $56.24 \%$, se vio incrementada en un $26,96 \%$ dando como resultado una media final de $83.2 \%$.

Por último, cabe destacar que, debido a la falta de tiempo y de personal, no fue posible entrevistar a los alumnos ni pedirles que rellenaran encuestas sobre su opinión respecto a las prácticas comunicativas y al impacto en el conocimiento de su entorno inmediato y de los servicios que ofrece. Sin embargo, dada la satisfacción mostrada por los alumnos con respecto a las mismas, interpretamos que resultaron funcionales, prácticas y que cumplieron con su objetivo inicial: acercar a los alumnos a su nuevo contexto, facilitarles el conocimiento de su nuevo entorno, facilitarles oportunidades de utilizar los contenidos aprendidos en el aula en un contexto real de comunicación y, en definitiva, favorecer a su inclusión social.

Como trabajo futuro, esperamos que en el próximo verano se pueda aplicar el material en dos grupos, de forma que en uno se llevarían a cabo las prácticas comunicativas y en otro, no. Así, tendremos la oportunidad de contrastar los resultados de ambos grupos y valorar el impacto de las prácticas comunicativas en la mejora lingüística de los alumnos y en su conocimiento sobre su entorno inmediato y los servicios que tienen a su alcance. A su vez, se prevé la creación de una rúbrica de evaluación y una ficha de evaluación para que los estudiantes valoren las prácticas comunicativas.

\section{Bibliografía}

Canale, M., \& Swain, M. (1980). «Theoretical bases of com-municative approaches to second language teaching and testing», Applied linguistics, 1, 1-47.

GARcíA, P. (1997). «El espacio comunicativo: Entorno de enseñanza y aprendizaje para inmigrantes». En F. Moreno, M. Gil y K. Alonso (eds.); La Enseñanza del Español como Lengua Extranjera: del Pasado al Futuro. Actas del VIII Congreso Internacional de 
ASELE. Alcalá de Henares, 17-20 de septiembre de 1997 (pp. 357-362). Alcalá de Henares: Universidad de Alcaláde Henares.

Del Estal Villarino, M. G. (2008). «La enseñanza de la gramática en el aula de español como L2 para el alumnado inmigrante», I Jornadas sobre Lenguas, Currículo y Alumnado Inmigrante, 83- 94.

Di FrANCO, C. (2006). «La enseñanza de la expresión oral en la clase de ELE: El español coloquial». En Gimeno, A. M. (ed.); Las destrezas orales en la enseñanza del español L2-LE. Actas del XVII Congreso Internacional de ASELE (pp. 469-482). Logroño: Universidad de La Rioja.

HAYAS, K. M. (2009). «Algunas propuestas didácticas actuales sobre la enseñanza del léxico: para un mejor tratamiento del aprendizaje del vocabulario», El currículo de ELE en Asia-Pacifico (pp. 353 - 367). Manila: Instituto Cervantes.

LÓPEZ, L. M. (1995). «Reflexiones previas sobre la enseñanza de E/LE a inmigrantes y refugiados», Didáctica (lengua y literatura), 7, 241-254.

Menegotto, A. (1998). «Del aula a la calle: La integración de objetivos y la transferencia de habilidades en el programa Mar del Plata», Español como Lengua Extranjera: Enfoque Comunicativo y Gramática. Actas del IX Congreso Internacional de ASELE. Santiago de Compostela, 863-873.

SALAZAR, V. (1992). «Gramática y enseñanza comunicativa del español-lengua extranjera», MarcoELE, 2, 1-34.

STARC, M. K. (2016). «El desarrollo de la competencia sociolingüística en la clase de español como lengua extranjera», Actas de las primeras Jornadas de Jóvenes Lingüistas. Buenos Aires: Universidad de Buenos Aires. 